\title{
Cathodoluminescence characterization of dilute nitride GaNSbAs alloys
}

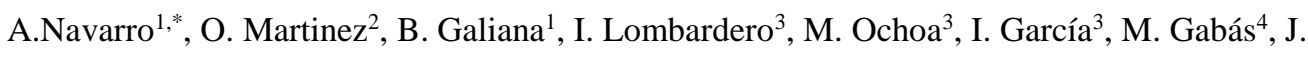 \\ Jimenez $^{2}$, C. Algora $^{3}$, C. Ballesteros ${ }^{1}$.
}

Corresponding author: amnavarr@fis.uc3m.es

${ }^{1}$ Physics Department, Universidad Carlos III de Madrid, Av. Universidad 40, 28911, Leganés, Madrid (Spain)

${ }^{2}$ GdS-Optronlab group, Dpto Física de la Materia Condensada, Univ. de Valladolid, Edificio LUCIA, Paseo de Belén 19, 47011 Valladolid (Spain)

${ }^{3}$ Instituto de Energía Solar, UPM. Av Complutense s/n 28040, Madrid (Spain)

${ }^{4}$ The Nanotech Unit, Dpto Física Aplicada I, Universidad de Málaga, 29071, (Spain)

\begin{abstract}
$\underline{\text { Abstract }}$
The effects of ex-situ annealing in $\mathrm{N}$ ambient and in-situ annealing in As ambient on GaNSbAs/GaAs structures grown by molecular beam epitaxy (MBE) were investigated by low temperature cross-sectional cathodoluminescence (CL). The amount and distribution of Sb was measured by energy dispersive spectroscopy (EDS). The cross-sectional CL analysis of all samples reveals a shift of the near band edge (NBE) emission along the growth axis, presumably associated with a non-uniform incorporation of Sb during the growth process, in agreement with the Sb distribution measured by EDS in the as-grown sample. The NBE emission in the annealed samples presents a redshift with respect to the as-grown sample. This effect might be explained by a redistribution/activation of $\mathrm{N}$ in the GaNSbAs lattice since the $\mathrm{Sb}$ distribution measured by EDS did not reveal significant changes, within the error margin, with respect to the as-grown sample. The in-situ annealed in As overpressure sample shows the best properties for solar cells applications, i.e., a NBE peak position close to 1.0 $\mathrm{eV}$ and the lowest full width at half maximum of this emission.
\end{abstract}

\section{$\underline{\text { Keywords }}$}

Dilute nitrides, GaNSbAs, Solar cells, Cathodoluminescence, Annealing.

\section{$\underline{\text { Introduction }}$}

In recent years diluted III-N-V semiconductor alloys have attracted a great deal of attention. Most of the research effort has been devoted to $\mathrm{GaIn}(\mathrm{N}) \mathrm{As}(\mathrm{Sb})$ alloys because the incorporation of a small amount of $\mathrm{N}$ can substantially reduce the bandgap, while preserving the lattice matching to GaAs. This is relevant for III-V 
multijunction solar cells. In particular, the current world record efficiency is $44 \%$ with a three junction $\mathrm{GaInP/GaAs} / \mathrm{GaInNAs}(\mathrm{Sb})$ structure grown on GaAs [1]. However, the incorporation of $\mathrm{N}$ into GaInNAs is a challenging issue, since structural defects are easily generated; as a consequence, the layer electrical properties are degraded due to either native point defects with deep electronic levels in the bandgap [2], or to N-related defects [3]. For this reason a considerable interest has been devoted to the development of alternative quaternary dilute nitrides, such as GaNSbAs, which can also be grown lattice-matched to GaAs with a bandgap of $1 \mathrm{eV}$. This compound presents better thermal stability due to the fact that it contains just only one element of group III, and needs lower $\mathrm{N}$ concentration than GaInNAs to reach the same bandgap reduction [4]. As it happens with other dilute nitrides, post-growth annealing of GaNSbAs has been revealed beneficial to achieve a good quality material; however, the reason for this improvement is still not well understood [5-10]. Some authors have studied the effects of the thermal annealing by photoluminescence (PL) combined with high resolution X-ray diffraction (HRXRD) and secondary ion mass spectroscopy (SIMS) [7, 9, 10]; others have studied this effect by spectroscopic ellipsometry (SE) together with HRXRD and SIMS [8]. Also, PL and photoreflectance spectroscopy (PR) [6] or PL together with HRXRD and transmission electron microscopy (TEM) have been used [5]. Regarding the use of cathodoluminescence (CL) in the study of dilute nitride GaNSbAs properties, plan view studies have been reported $[11,12]$. However, the high spatial resolution of the CL should allow to achieve cross sectional analysis of the GaNSbAs layer, which to the best of our knowledge has not been previously reported. In this work, we present a detailed investigation on the effects of $e x$-situ annealing under $\mathrm{N}$ ambient and insitu annealing under As ambient by cross-sectional cathodoluminescence (CL) to have a deeper understanding of the relation between the improvement of the optical properties and the annealing processes. The main benefit of the CL technique is its high spatial resolution that allows studying the luminescence emission across the dilute nitride layer, providing information about the composition gradients of the GaNSbAs layers along the growth axis, with spatial resolution around $100 \mathrm{~nm}$. The $\mathrm{CL}$ results are complemented with scanning transmission electron microscopy (STEM) and energy dispersive spectroscopy (EDS) measurements.

\section{Experimental}

Epilayers were grown on (001) GaAs substrates by molecular beam epitaxy (MBE) using a radio frequency (RF) plasma-assisted nitrogen source, and a valved antimony cracker source. The samples were grown at an RF plasma 
power of $180 \mathrm{~W}$, and substrate temperature of $500^{\circ} \mathrm{C}$. The $\mathrm{Sb}$ beam equivalent pressure and the As/Ga beam equivalent pressure ratio allowed the growth of GaNSbAs layers with $2 \% \mathrm{~N}$ and $6 \% \mathrm{Sb}$, as estimated by high resolution x-ray diffraction (HXRD) measurements [13]. The samples analyzed are complete GaNSbAs solar cell

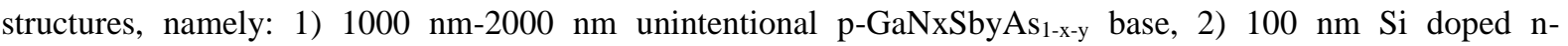
$\mathrm{GaN}_{\mathrm{x}} \mathrm{Sb}_{\mathrm{y}} \mathrm{As}_{1-\mathrm{x}-\mathrm{y}} / 100 \mathrm{~nm}$ Si doped n-GaAs emitter, 3) $50 \mathrm{~nm}$ n+AlGaAs window layer and 4) 50-500 nm n+GaAs contact layer. Since the p-GaNxSbyAs 1-x-y $_{\text {b }}$ base is unintentional doped, the doping level is expected to be below $10^{16} \mathrm{~cm}^{-3}$.

Three samples were studied: a) an as-grown sample with a $2000 \mathrm{~nm}$ thick base layer, thereafter labeled as asgrown. b) a postgrowth ex-situ annealed sample in $\mathrm{N}$ ambient at $750^{\circ} \mathrm{C}$ for 5 minutes, labeled as ex-situ annealed; and c) an a post-growth in-situ annealed sample under As overpressure at $700^{\circ} \mathrm{C}$ for 5 minutes, labeled as in-situ annealed.

The CL measurements were carried out in cross-section with a Gatan MonoCL2 system attached to a LEO 1530 field emission scanning electron microscope (FESEM). The panchromatic CL (panCL) images were obtained using a single channel InGaAs detector (800-1800 nm detection range). The detection for spectrally resolved CL was done with a Peltier cooled Si CCD camera (200-1100 nm spectral range). The CL spectra of the GaNSbAs layers were sequentially collected from the GaAs substrate to the AlGaAs window layer by scanning in steps of

口 $100 \mathrm{~nm}$. The CL spectra were obtained at $80 \mathrm{~K}$ in order to improve the signal to noise ratio and at $5 \mathrm{kV}$ to ensure a high lateral resolution of the order of $100 \mathrm{~nm}$.

Cross section chemical profiles were measured by EDS in a Philips Tecnai F20 Transmission Electron Microscope (TEM) with a Scanning Transmission (STEM) module and High Angle Annular Detector (HAAD) for Z-contrast imaging operated at $200 \mathrm{keV}$.

\section{$\underline{\text { Results and discussion }}$}

The cross section panCL images of the as-grown, ex-situ annealed ( $\mathrm{N}$ atmosphere) and in-situ annealed (As atmosphere) samples are displayed in Figures 1a-1c. All samples reveal a GaNSbAs layer with dark contrast, implying a lower luminescence emission than the GaAs substrate. In the as-grown and ex-situ annealed samples, a bright layer with irregular contrast corresponding to the GaAs $(n+)$ doped contact layer is observed. The CL emission of this layer is weak in the in-situ annealed sample because of its small thickness $(\sim 50 \mathrm{~nm})$. 
Figures $2 \mathrm{a}-2 \mathrm{c}$ show the CL spectra consecutively acquired in steps of $\mathrm{Q} 100 \mathrm{~nm}$ along the growth axis of the GaNSbAs layer for the as-grown, ex-situ and in-situ annealed samples, respectively. For each spectrum, the main peak has been identified as the near band edge (NBE) emission, which will follow a similar evolution to the bandgap, even if one cannot assert that the dominant peak of the NBE emission is the conduction band (CB) to valence band (VB) transition. For each set of spectra, we have calculated the relative NBE peak shift, labeled $\Delta \mathrm{E}$, defined as the difference between the specific NBE energy for each spectrum and the reference value for this peak, set as the value measured in the spectrum collected in the first $100 \mathrm{~nm}$ of GaNSbAs layer. These data ( $\Delta \mathrm{E}$ vs. GaNSbAs layer depth) are plotted in Figure 2d for the three samples showing the NBE peak shift profile across the layer.

Analyzing Figure 2a the as-grown sample presents a CL peak around $1.147 \mathrm{eV}$, corresponding to the NBE emission of GaNSbAs, which shifts to higher energies along the growth direction. This variation ( $\triangle \mathrm{E}$ vs. GaNSbAs layer depth), plotted in Figure 2d, shows an almost linear behavior, which increases from $1.137 \mathrm{eV}$ (bottom of the layer, $100 \mathrm{~nm}$ ) to $1.153 \mathrm{eV}$ (top of the layer, $2000 \mathrm{~nm}$ ). The resultant NBE shift per unit length of the GaNSbAs layer calculated as $\Delta \mathrm{E} / W$, being $W$ the total thickness of the layer, is $8 \cdot 10^{-3} \mathrm{meV} / \mathrm{nm}$. Considering that the NBE peak energy is directly related to the concentrations of $\mathrm{N}$ and/or $\mathrm{Sb}$ in the material, this shift can be assimilated to a composition gradient along the growth direction. In particular, since the NBE peak energy should increase as N and/or Sb decrease [14], one can argue about a reduction of the incorporation rate of $\mathrm{Sb}$ and/ or $\mathrm{N}$ along the growth direction. In fact, the surfactant nature of $\mathrm{Sb}$ enhances the $\mathrm{N}$ incorporation in dilute nitrides [15], so, an inhomogenous incorporation of Sb may have a concomitant influence on the $\mathrm{N}$ distribution in the GaNSbAs layer. Regarding the ex-situ annealed sample (Figure 2b) the CL spectra reveal a global decrease of the NBE peak position (red-shift), in relation to the as-grown sample, with NBE energy around $1.125 \mathrm{eV}$. For this sample, the NBE energy also exhibits a shift towards higher energies along the growth direction, particularly from $1.111 \mathrm{eV}$ (at the lower interface, $100 \mathrm{~nm}$ ) to $1.132 \mathrm{eV}$ (at the top of the layer, $2000 \mathrm{~nm}$ ), although, in this case, the behavior is non-linear, contrarily to the as-grown sample. The difference between the maximum and the minimum NBE energy divided by the layer thickness $(W)$ was calculated to achieve an average energy shift per unit length of the GaNSbAs. The estimated value was $\Delta \mathrm{E} / W=9 \cdot 10^{-3} \mathrm{meV} / \mathrm{nm}$.

Additionally, in Figure $2 \mathrm{~b}$ the spectra present an additional defect related band, around $1.05 \mathrm{eV}$ in the low energy tail of the NBE peak, which appears in the upper part of the GaNSbAs layer (from $400 \mathrm{~nm}$ to $2000 \mathrm{~nm}$ ) and whose intensity increases along the growth direction. This wide band could be related to a loss of As or/and to an 
increment in the $\mathrm{N}$ content in the GaNSbAs layer due to the annealing process in $\mathrm{N}$ ambient [7], which would cause an As desorption and a $\mathrm{N}$ incorporation at such high temperature. Both hypotheses are coherent with the fact that the intensity of this low energy band increases closer to the surface.

Finally, in the in-situ annealed sample (Figure 2c) the CL spectra present a global decrease (redshift) of the NBE energy (around $1.116 \mathrm{eV}$ ) compared to both the as-grown and the ex-situ annealed samples. Similar to the ex-situ annealed sample, the NBE shift was not linear along the growth axis. It shifted from $1.113 \mathrm{eV}$ (lower interface, $100 \mathrm{~nm}$ ) to $1.119 \mathrm{eV}$ (top of the layer, $1000 \mathrm{~nm}$ ) which results in a NBE shift per unit length $(\Delta \mathrm{E} / \mathrm{W})$ equal to $6 \cdot 10^{-3} \mathrm{meV} / \mathrm{nm}$. In this sample, the defect related band observed in the ex-situ annealed sample does not appear. The non-existence of this band suggests that in the in-situ annealing the loss of As is suppressed, as a result of the As overpressure during the thermal annealing which avoids the As desorption, being consistent with the results achieved for the $e x$-situ annealed sample.

The optical quality of the three samples has been analyzed comparing the NBE peak intensity of the spectra recorded at $500 \mathrm{~nm}$ depth and the full width at half maximum (FWHM) of the NBE peak as a function of the layer depth. The data are displayed in Figure 3. It can be observed that the intensity in the in-situ sample is higher than for the two other samples, while the FWHM of the NBE peak is narrower than the ones measured for the other samples for the same GaNSbAs layer depth. In fact, for the $e x$-situ sample the FWHM increases at the point where the additional low energy band appears in the CL spectra, indicating a relation between the NBE band broadening and the secondary band emergence, which points to an increase of defect concentration under the annealing in $\mathrm{N}_{2}$ atmosphere.

The CL results were complemented by cross section EDS profiles along the growth axis of the GaNSbAs layer. Figure 4a shows the STEM image of the as-grown sample, where the red arrow indicates the EDS scan line location. This image shows flat and sharp top and bottom GaNSbAs interfaces. The STEM images of the ex-situ and in-situ annealed samples (not shown here) exhibited a similar morphology as the asgrown sample; therefore, one can argue that the annealing process does not modify the layer morphology or the interfaces quality. Additionally, the homogenous STEM contrast in the GaNSbAs layer accounts for the absence of significant $\mathrm{Sb}$ or Ga clustering. In the upper parts of figures $4 \mathrm{~b}$ and $4 \mathrm{c}$ are plotted the NBE emission peak energy profiles along the growth direction of the GaNSbAs layers of the as-grown and exsitu annealed samples respectively. In the bottom part of Figure $4 \mathrm{~b}$ and $4 \mathrm{c}$, the profiles of $\mathrm{Sb}$ in the growth direction for the same layers, achieved by EDS measurements, are shown. Nitrogen is not included since with the resolution achieved it cannot be clearly identified by EDS. In fact, electron energy loss spectroscopy (EELS) measurements were carried out, which is the 
most appropriate technique for detecting light elements, but as a result of the low $\mathrm{N}$ content ( $\square 2 \%$ ), nitrogen could not be properly detected. The compositions presented in atomic percentage are semi-quantitative measurements, which are achieved by fitting the experimental data. This procedure avoids the effect of the layer thickness since it calculates the element percent for each isolated spectrum. It is important to remark that the values estimated for Sb have an error of $\square 2 \%$, due to the low Sb content. Consequently, they are mere indicative values, i.e, they are useful to evaluate the tendency of the elements distribution across the layer under study.

The monotonous increase of the NBE peak energy along the growth axis of the as-grown sample can be related to the increase of the band gap energy (Figure $4 b$ ). This result might be explained by the decrease of the EDS Sb signal intensity along the growth direction observed in the bottom of Figure $4 b$, since the GaNAsSb bandgap energy increases as Sb decreases. Consequently, one might speculate about the role of $\mathrm{Sb}$ in the bandgap energy profile of the as-grown sample.

Regarding the annealed samples, the overall redshift of the NBE peak with respect to the as-grown sample can be due either to an increase in the Sb concentration or to the activation of N. The EDS data of the exsitu sample of figure $4 \mathrm{c}$ do not reveal a relevant increase of $\mathrm{Sb}$ with respect to the as-grown sample, within the error margins; therefore, the redshift of the NBE emission cannot be related to a Sb increase. Additionally, for the ex-situ annealed sample some As loss or $\mathrm{N}$ increase could be expected, which could account for the redshift of the NBE peak. Conversely, in the case of the in-situ annealed sample, the As overpressure could increase the As concentration, resulting in a relative decrease of the $\mathrm{Sb}$ atomic fraction, which would cause a blueshift of the NBE peak. Therefore, the redshift measured must be associated with the activation of N. Consequently, both thermal treatments seem to act on the effective activation of $\mathrm{N}$, since they induce a redshift on the NBE peak and relevant modifications on Sb content along the growth direction are not detected.

Previous reports on annealed GaNSbAs have reported blueshift of the NBE luminescence emission, while in this work, we have observed redshift after annealing. This result has been reported for other dilute nitrides, e.g. GaNInAs, for which both blueshift $[16,17]$ and redshift $[18,19]$ have been reported to occur under annealing. Both effects, blueshift and redshift, seem to be associated with the distribution/activation of N. In particular, the blueshift in GaNInAs was related to the N motion under annealing, the Ga-N bonds of the as-grown samples are transformed in In-N bonds by the annealing $[16,17]$. For GaNSbAs it was claimed that the $\mathrm{N}$ pairs thermally dissociate forming substitutional $\mathrm{N}$ [6]. Others authors argued that the redshift is related to defect levels in the band gap associated with N-related complex defects [7]. The redshift in GaNInAs is associated with $\mathrm{N}$ interstitials 
entering in substitutional sites by annealing $[18,19]$. This seems coherent with our results, that point to a redshift related to the activation of $\mathrm{N}$ rather than to significant changes in the $\mathrm{Sb}$ composition.

Comparing the different samples, it can be noticed that the annealing process under As overpressure (insitu annealed sample) presents a higher redshift, getting closer to our $1 \mathrm{eV}$ bandgap target, the lowest FWHM as well as the smallest energy bandgap shift along the growth direction. Our results are coherent with previous studies, where it was reported that the PL characteristics of the in-situ annealed samples (As atmosphere) were better than those of the ex-situ annealed samples $[7,9,10]$. The reason argued for this improvement was a more efficient elimination of $\mathrm{N}$-related recombination centers $[9,10]$, as well as a suppression of both $\mathrm{N}$ and As out-diffusion that leads to an improvement of Ga-N bonding [7]. In fact, the improvement of the optical properties of the in-situ annealed sample has been related to a nitrogen redistribution as a consequence of the stabilization of the As lattice $[7,9,10]$.

\section{Conclusions}

GaNSbAs epilayers lattice matched to GaAs grown by MBE have been characterized by cross-sectional CL analyzing the shift of the NBE peak energy across the layer. EDS measurements have also been carried out for chemical characterization. Whereas EDS does not inform about $\mathrm{N}$ gradients, CL profiles along the growth direction allows drawing a picture of the band gap shift in the three studied samples. In all the three samples we have observed an increase of the NBE peak energy along the growth axis. While this energy shift is related to a $\mathrm{Sb}$ gradient in the as-grown sample, the overall redshift observed in the annealed samples renders a relevant role to the activation of nitrogen by the annealing processes. This redshift seems to be mainly related to the redistribution/activation of $\mathrm{N}$ in the lattice of GaNSbAs annealed samples. Finally, comparing all the samples, the in-situ annealing process has revealed itself to present the best properties (lowest FWHM of the NBE peak and the closest value to $1 \mathrm{eV}$ ) for solar cell applications. Further work is needed to correlate the $\mathrm{Sb}$ and/or $\mathrm{N}$ distribution with the NBE redshift. Cross sectional CL has been shown to be a powerful, simply and nondestructive tool to study GaNAsSb layers and very promising for analyzing qualitatively the $\mathrm{N}$ distribution in III$\mathrm{N}-\mathrm{V}$ dilute nitrides.

\section{Acknowledgements}

O.Martínez and J. Jimenez were funded by Spanish Government (MINECO project ENE2014-56069-C44-R) and Junta de Castilla y León (VA293U13 and VA081U16 projects). The Ministry of Economy and 
This is a pre-print of an article published in Journal of Electronic Materials, 2018, Volume 47, Issue 9, pp 5061-5067. The final authenticated version is available online at: https://doi.org/10.1007/s11664-018-6325-3

Competitiveness MINECO supports this work through projects TEC2014-54260-C3-1-P, TEC201454260-C3-2P, TEC2014-54260-C3-3-P, PCIN-2015-181-C02-01 and PCIN-2015-181-C02-02.

\section{$\underline{\text { References }}$}

[1] R. Jones-Albertus, E. Becker, R. Bergner, T. Bilir, D. Derkacs, O. Fidaner, D. Jory, T. Liu, E. Lucow, P. Misra, E. Pickett, F. Suarez, A. Sukiasyan, T. Sun, L. Zhang, V. Sabnis, M. Wiemer, and H. Yuen, in Proc. MRS 1538, 161 ( 2013).

[2] P.Krispin, V. Gambin, J. S. Harris and K. H. Ploog, J. Appl. Phys. 93, 10 (2003).

[3] J-C. Harmand, A. Caliman, E. V. K. Rao, L. Largeau, J. Ramos, R. Teissier, L. Travers, G. Ungaro, B. Theys and I. F. L. Dias. Semicond. Sci. Technol. 17, 778 (2002).

[4] V. Braza, D. F. Reyes, A. Gonzalo, A. D. Utrilla, T. Ben, J. M. Ulloa and D. González, Nanoscale Res. Lett. $12,356(2017)$.

[5] D. P. Xu, J. Y. T. Huang, J. Park, L. J. Mawst, T. F. Kuech, X. Song and S. E. Babcock, Appl. Phys. Lett., 91, 191909 (2007).

[6] Y-T. Lin, T-C. Ma, H-H. Lin, J-D. Wu, and Y-S. Huang, Appl. Phys. Lett. 96, 011903 (2010).

[7] S. Bharatan, S. Iyer, K. Nunna, W. J. Collis, K. Matney, J. Reppert, A. M. Rao and P. R. C. Kent, J. Appl. Phys. 102, 023503 (2007).

[8] N. BenSedrine, X.Lafosse, O.Mauguin, R.Chtourou and J.C.Harmand, Mater Sci Semicond Process. 29, $331(2015)$

[9] J. Li, S. Iyer, S. Bharatan, L. Wu, K. Nunna, W. Collis, K. K. Bajaj and K. Matney, J. Appl. Phys. 98,013703 (2005)

[10] L. Wu, S. Iyer, K. Nunna, J. Li, S. Bharatan, W. Collis and K. Matney, J. Cryst. Growth 279, 293 (2005).

[11] L.Pelosi, G. Attolini, M. Bosi, M. Avella, M. Calicchio, N. Musayeva and J. Jiménez, J. Cryst. Growth 287, 625 (2006).

[12] J. Miguel-Sánchez, A. Guzmán, U. Jahn, E. Luna and E. Muñoz, Microelectron. J. 37, 1552 (2006). [13] K.H. Tan, S.Wicaksono, W.K.Loke a, D.Li, S.F.Yoon, E.A.Fitzgerald, S.A.Ringel and J.S.Harris Jr., J. Cryst. Growth 335, 66 (2011).

[14] C-Z. Zhao, H-F. Guo, T. Wei, S-S. Wang and K-Q. Lu, Physica B Condens Matter 485, 35 (2016).

[15] Yuen HB, Bank SR, Bae H, Wistey MA and Harris JS Jr., J. Appl. Phys 99, 093504 (2006). 
This is a pre-print of an article published in Journal of Electronic Materials, 2018, Volume 47, Issue 9, pp 5061-5067.

The final authenticated version is available online at: https://doi.org/10.1007/s11664-018-6325-3

[16] K. Volz, T. Torunski, O. Rubel, and W. Stolz, J. Appl. Phys. 104, 053504 (2008).

[17] K. Müller, M. Schowalter, A. Rosenauer, D. Hu, D. M. Schaadt, M. Hetterich, P. Gilet, O. Rubel, R.

Fritz and K. Volz, Phys. Rev. B 84, 045316 (2011).

[18] K. Gao, S. Prucnal, W. Skorupa, M. Helm and S. Zhou, Appl. Phys. Lett. 105, 012107 (2014).

[19] W. K. Cheah, W. J. Fan, S. F. Yoon, B. S. Ma, T. K. Ng, R. Liu and A. T. SWee, Semicond. Sci.

Technol. 21, 6 (2006). 

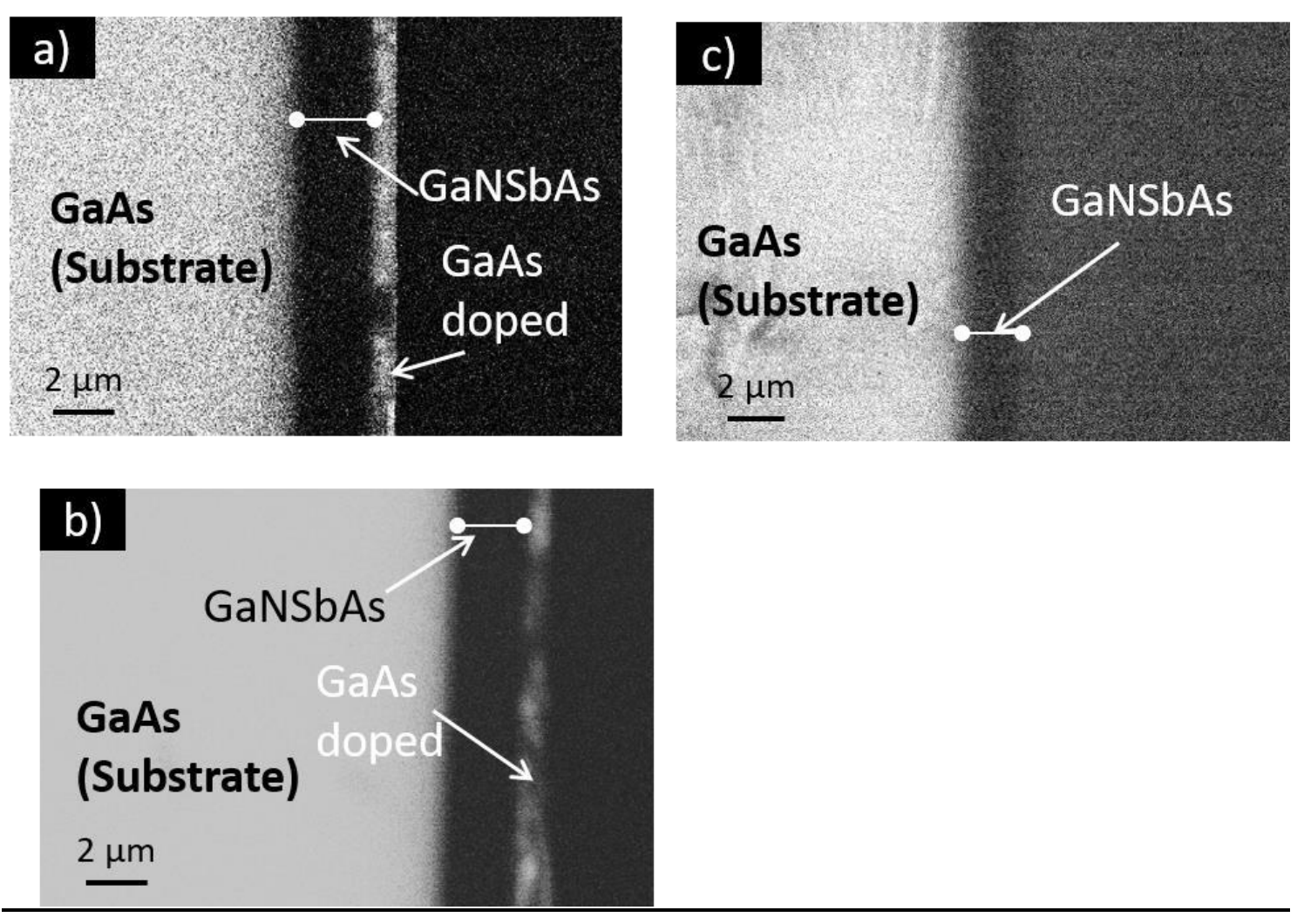

Figure 1. PanCL images in cross-section of the samples a) as-grown, b) ex-situ annealed subjected to $\mathrm{N}$ ambient at $750^{\circ} \mathrm{C}$ and c) in-situ annealed subjected to As ambient at $700^{\circ} \mathrm{C}$. 

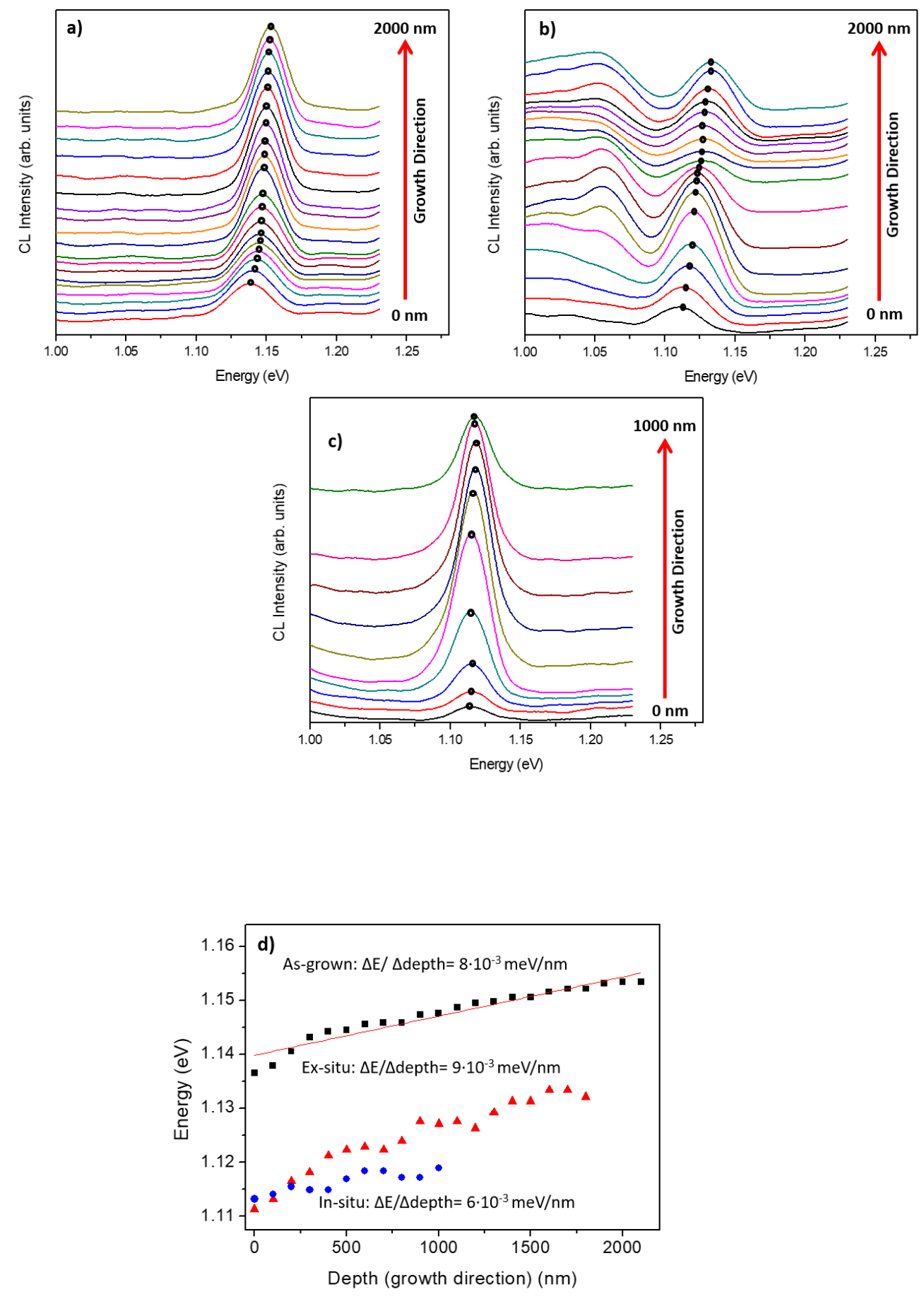

Figure 2. Cross-section CL spectra for a) as-grown, b) ex-situ annealed subjected to $\mathrm{N}$ ambient at $750^{\circ} \mathrm{C}$, 
c) in-situ annealed subjected to As ambient at $700^{\circ} \mathrm{C}$ (the spectra were acquired in steps of $100 \mathrm{~nm}$ across the layer), and d) NBE energy of the GaNSbAs layers of the corresponding samples (as-grown, ex-situ annealed and in-situ annealed) as a function of the depth in the growth direction.
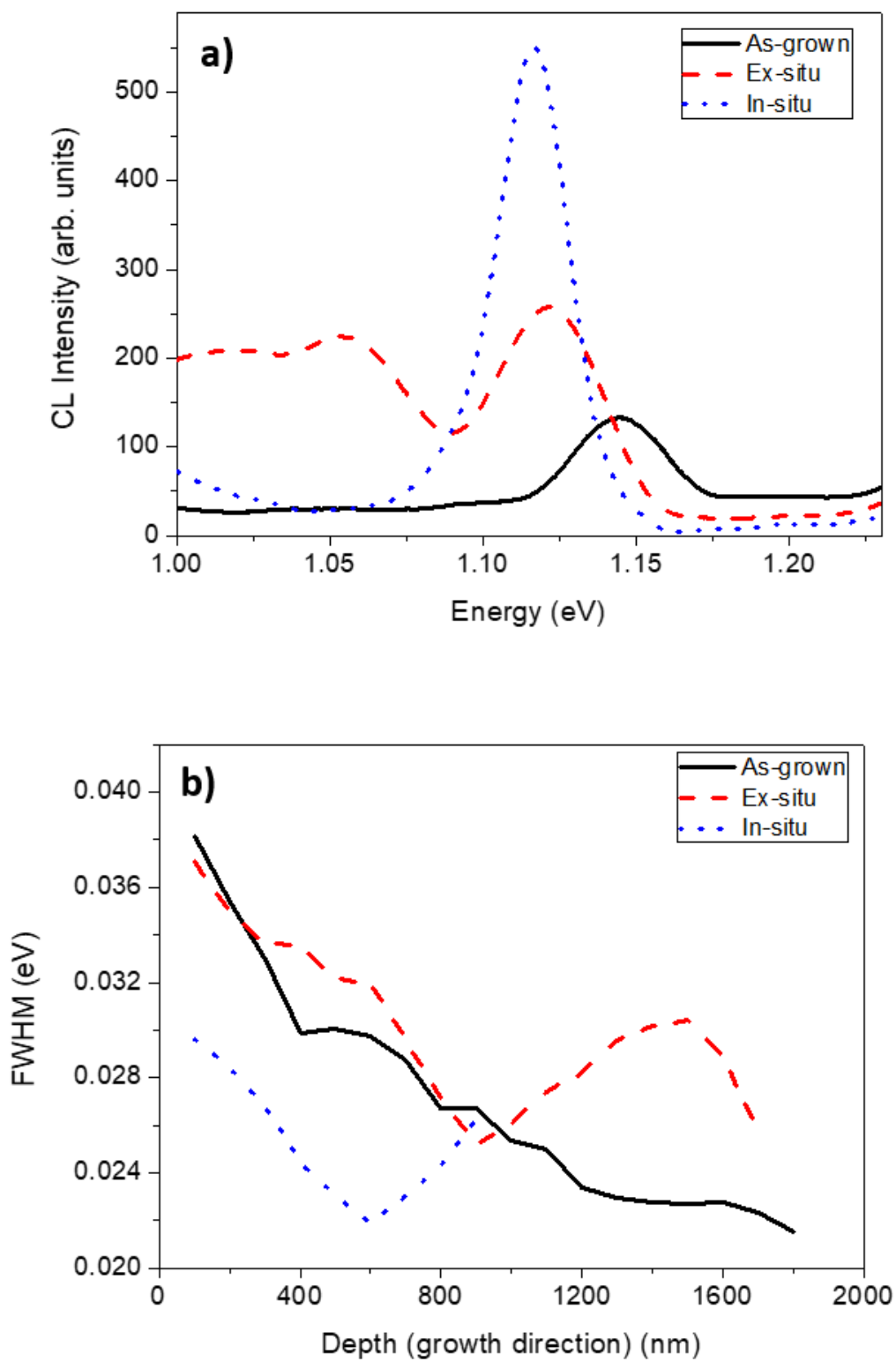

Figure 3. a) NBE peak intensity of the spectra recorded at $500 \mathrm{~nm}$ depth for the as-grown and the in-situ and exsitu annealed samples and b) full width at half maximum (FWHM) of the NBE peak as a function of depth for the three samples. 
This is a pre-print of an article published in Journal of Electronic Materials, 2018, Volume 47, Issue 9, pp 5061-5067.

The final authenticated version is available online at: https://doi.org/10.1007/s11664-018-6325-3
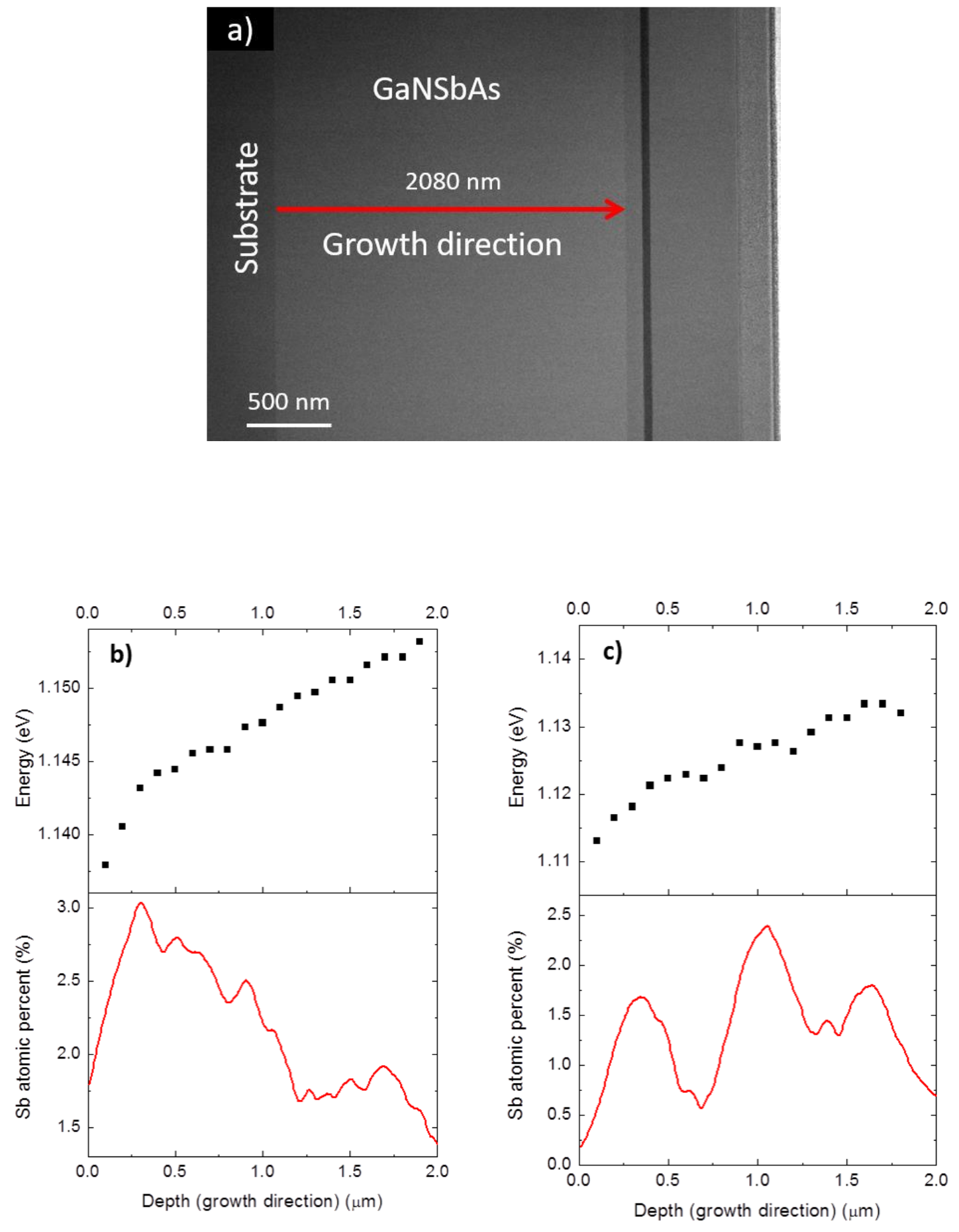
This is a pre-print of an article published in Journal of Electronic Materials, 2018, Volume 47, Issue 9, pp 5061-5067. The final authenticated version is available online at: https://doi.org/10.1007/s11664-018-6325-3

Figure 4. a) STEM-High angular annular dark field (HAADF) image of the as-grown sample. The arrow indicates the zone where the EDS profile was acquired. b) and c) (top) NBE peak intensity of the GaNSbAs layer of asgrown and ex-situ annealed samples, respectively, as a function of depth along the growth direction (CL data), and (bottom) semi-quantitative composition profiles of Sb obtained by EDS line scan along the growth direction of the as-grown, and ex-situ annealed samples. 